\title{
In celebration of chemistry
}

\author{
The year 2019 marks two noteworthy anniversaries in chemistry. We use this opportunity to reflect on the \\ importance of chemistry to Nature Methods and to the broader life science research community.
}

T o celebrate the 150th anniversary of Mendeleev's development of the periodic table, UNESCO has dubbed 2019 the International Year of the Periodic Table of Chemical Elements. IUPAC, the International Union of Pure and Applied Chemistry, is also celebrating a milestone: for a century now, it has served as the authority on chemical nomenclature and terminology, providing a common language for chemists to speak.

Chemistry has been called the "central science" because molecular structure and chemical transformations underpin other major fields of research, including materials science, nanotechnology, and, especially, biology. Because chemistry is so fundamental to these fields, the lines between the disciplines can blur, and it has become increasingly difficult to define exactly what the word "chemistry" means. An organic chemist is likely to come up with a different answer than a physical chemist, and a chemical biologist might not agree with either of their definitions.

At its most basic, chemistry can be defined in terms of the elements-their properties, how they interact and combine to form different substances. Mendeleev featured 63 known elements in his original periodic table, with gaps for elements that at that time had not yet been discovered; the periodic table today contains 118 known elements. Though the number of chemical elements involved in biological systems is of course relatively small, and though many life scientists might not think about chemistry on a daily basis, it is imperative to remember that chemical structure and reactivity are responsible for all biological processes at heart. Biological macromolecules-proteins, nucleic acids, lipids, carbohydrates-are simply supersized organic molecules. Metal ions are ubiquitous in biology and essential for the structure and function of numerous biomolecules. Small-molecule metabolites are pervasive, and yet still no one knows how many metabolites are present at a particular point in time or what all of their structures are. The number of chemical transformations occurring at any given moment in an organism is mind-bogglingly enormous.

Chemists must do their part to accept that the boundaries of what should be considered chemistry have grown, embrace this expansion, and support their colleagues who work at the interface of other fields. Many traditionally minded chemists grumble, for example, when Nobel Prizes in Chemistry are awarded for biologically oriented discoveries, such as ubiquitin-mediated protein degradation (2004), ribosome structure and function (2009), or mechanistic studies of DNA repair (2015). Chemists have made substantial contributions to tools and techniques considered to be in the realm of biology_including nucleic acid sequencing; fluorescent proteins and small-molecule probes; mass-spectrometrybased proteomics and metabolomics; and cryo-electron microscopy, nuclear magnetic resonance spectroscopy, X-ray crystallography, and other approaches in structural biology - and to the development of bio-orthogonal chemistries to probe molecular function in cells, to name just a few examples.

Biology needs basic chemistry research, just as translational research needs basic biology research. New advances for understanding and probing biology are likely to come from chemistry, as well as physics and computer science. Without chemists working to develop new kinds of reactions, without researchers with the skills to build new molecules and tinker with them to make better tools, and without continued development of analytical instrumentation, the life sciences will advance only so far. This is not to say that areas in the traditional realm of chemistry are no longer relevant. As chemist Phil Baran wrote of synthetic organic chemistry in a Journal of the American Chemical Society editorial last year, "As a community, we have become quite adept at being able to make anything with enough resources, but we are still decades or perhaps even centuries away

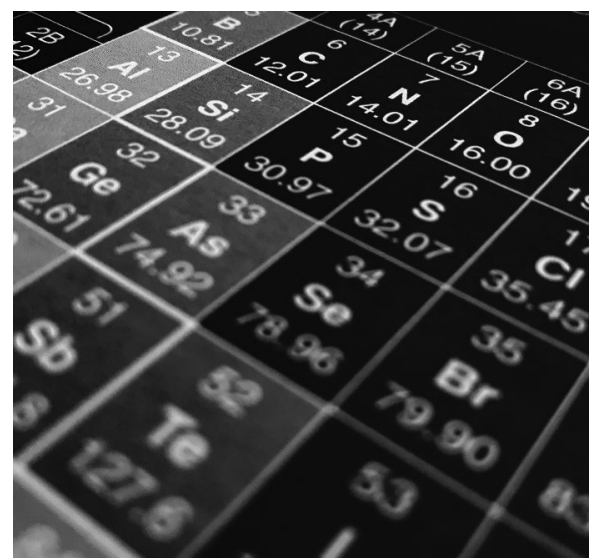

Credit: Allison Doerr

from making everything well." There is still much to do to advance chemical knowledge in its own right.

Here at Nature Methods, we remain committed to publishing tools and methods that advance basic research in the life sciences, from the molecular scale to the whole-organism level. Methods development is hard, and it takes skill, patience, and willingness to spend time doing tedious optimizations. Methods developers usually do not get the accolades that a flashy new biological finding will receive, but long-term impacts can be substantially more meaningful for advancing science. We hope that our readers, no matter what their area of life science research, will take a few minutes to reflect on how tools and methods rooted in chemistry have had a positive effect on their research.

We leave you with a few links to special features celebrating Mendeleev's achievement published in Nature (https:// www.nature.com/collections/daffidjhif) and Nature Chemistry (https://www.nature. com/collections/jksxfggtbd), including an interactive periodic table.

Published online: 30 May 2019 https://doi.org/10.1038/s41592-019-0454-3 